\title{
Homocysteine - relation to hypertension, age and smoking in patients with newly diagnosed essential hypertension
}

\author{
Katarzyna Korzeniowska, Artur Cieślewicz, Ewa Chmara, Anna Jabłecka \\ Department of Clinical Pharmacology, Poznan University of Medical Sciences, Poland
}

\begin{abstract}
Introduction. An elevated circulating concentration of homocysteine is associated with an increased risk of coronary, cerebral, and peripheral vascular disease. The purpose of this study was to determine the concentrations of homocysteine in the patients with newly diagnosed essential hypertension and healthy subjects and to analyze the correlation between homocysteine level and the other parameters (age, blood pressure, smoking).

Material and methods. The study group consisted of 18 patients with essential hypertension, 4 women and 14 men (mean age $43 \pm 16$ years) and 15 healthy volunteers, 8 women and 7 men (mean age $47 \pm 10$ years). Serum homocysteine was analyzed by FPIA method (Fluorescence Polarization Immunoassay).

Results. The patients with essential hypertension had significantly higher homocysteine concentration compared to control group. No correlation was observed between homocysteine levels and age, diastolic, systolic blood pressure in subjects with essential hypertension. In healthy volunteers, only a correlation between age and homocysteine concentration was found.
\end{abstract}

Keywords: homocysteine, hypertension, blood pressure.

\section{Introduction}

Homocysteine is a sulfur-containing intermediate product in the normal metabolism of methionine, an essential amino acid.

Homocysteine (Hcy) is a product of dietary methionine demethylation, an abundant aminoacid in animal protein. It is present in plasma in four different forms: around $1 \%$ circulates as free thiol, $70-80 \%$ remains disulphide-bound to plasma proteins, mainly albumin

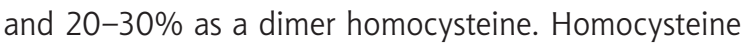
is a key determinant of the methylation cycle [1].

Measurement of total plasma or serum homocysteine represents the sum of oxidized and protein bound homocysteine. Homocysteine contains a reactive sulphydryl group that can react with plasma constituents, which may promote oxidative damage. An elevated homocysteine level therefore induces thrombogenicity, causes procoagulant state and promotes the proliferation of smooth muscle cells [2].
Hyperhomocysteinemia is characterized by an abnormally high level (above $15 \mu \mathrm{mol} / \mathrm{L}$ ) of homocysteine in the blood (Table 1); normal range for plasma homocysteine concentration increases with age (Table 2). Other factors influencing homocysteine concentration are genetic factors, drugs, clinical conditions (renal and thyroid dysfunction, cancer, psoriasis, diabetes), lifestyle (alcohol, tobacco, coffee), gender, menopause, muscle mass [3].

Two types of hyperhomocysteinemia, which can be distinguished, are:

- rare severe forms caused by major mutations of genes encoding enzymes responsible for homocysteine metabolism,

- more common moderately elevated homocysteine levels related to genetic and environmental factors [4].

Folic acid, vitamin B12, and B6 deficiency and reduced enzyme activities inhibit the breakdown of homocysteine, thus increasing the intracellular homocysteine concentration [5]. 
Table 1. Concentration of homocysteine in various stages of hyperhomocysteinemia

\begin{tabular}{cc}
\hline & Plasma homocysteine concentration $[\mu \mathrm{mol} / \mathrm{L}]$ \\
\hline Normal range: & \\
HPLC & $5.0-15.0$ \\
immunoassay & $5.0-12.0$ \\
\hline Moderate hyperhomocysteinemia & $16.0-30.0$ \\
\hline Intermediate hyperhomocysteinemia & $31.0-100.0$ \\
\hline Severe hyperhomocysteinemia & $>100.0$ \\
\hline
\end{tabular}

Table 2. Normal reference ranges of plasma homocysteine levels for different age groups [years]

\begin{tabular}{ccc}
\hline & Age 12-19 & Age $\geqslant 60$ \\
\hline Men & $4.3-9.9$ & $5.9-15.3$ \\
\hline Women & $3.3-7.2$ & $4.9-11.6$ \\
\hline
\end{tabular}

Numerous retrospective and prospective studies have consistently found an independent relationship between mild hyperhomocysteinemia and cardiovascular diseases or all-cause mortality [1].

Increase in homocysteine level can lead to damage of endothelial cells, decreased flexibility of blood vessels leading to aortic stiffness and to reduction of the speed of blood flow, reduced production of the vasodilator nitric oxide (NO). Therefore, increased plasma homocysteine can promote atherosclerotic disease, including coronary disease, stroke and peripheral vascular disease.

Several epidemiological studies revealed that a 5 $\mu \mathrm{mol}$ increase in plasma homocysteine results in $60 \%$ higher prevalence of ischaemic heart disease. Other studies demonstrated that the treatment of hyperhomocysteinaemia reduces atherosclerotic plaque area, thus decreasing the risk of deep vein thrombosis, stroke and ischaemic heart disease [6-7].

High blood pressure is a major risk factor for cardiovascular diseases. Although its etiology has not been fully explained mostly because of as yet unknown genetic variation, multiple nonhereditary factors including dietary and other lifestyle factors have been identified to have important and modifiable influences on blood pressure. Results of several studies suggest that mild increase in serum homocysteine may contribute to elevations in blood pressure [8].

The hypothesis that homocysteine may play a role in the pathogenesis of essential hypertension is based on the fact that homocysteine induces arteriolar constriction, renal dysfunction, increased sodium reabsorption and arterial stiffness. Also, elevated homocysteine is known to increase oxidative stress that causes oxidative injury to the vascular endothelium, diminishes vasodilation by nitric oxide, stimulates the proliferation of vascular smooth muscle cells, and alters the elastic properties of the vascular wall. All these factors are associated with the rise in hypertension. Thus, homocysteine may contribute to blood pressure elevation [9-12].

Little is known about the relation between homocysteine levels and blood pressure in newly diagnosed essential hypertension. Therefore, we have tested the homocysteine concentration in relation to the age, blood pressure and smoking in the patients with newly diagnosed hypertension.

\section{Material and methods}

The study was carried out in the Department of Clinical Pharmacology (University of Medical Sciences

Table 3. Clinical characteristic of patients with newly diagnosed essential hypertension and control

\begin{tabular}{ccc}
\hline & Patients & Control \\
\hline Number of participants & $22(8 \mathrm{~F}, 14 \mathrm{M})$ & $18(9 \mathrm{~F}, 9 \mathrm{M})$ \\
\hline Age [years] & $43 \pm 16$ & $47 \pm 10$ \\
\hline Height $[\mathrm{cm}]$ & $174 \pm 11$ & $171 \pm 6$ \\
\hline Weight $[\mathrm{kg}]$ & $76 \pm 13$ & $70 \pm 9$ \\
\hline Body mass index $\left[\mathrm{kg} / \mathrm{m}^{2}\right]$ & $24.72 \pm 2.22$ & $23.21 \pm 1.86$ \\
\hline Smoking $[\mathrm{n}]$ & 12 & 10 \\
\hline SBP $[\mathrm{mm} \mathrm{Hg}]$ & $158 \pm 8$ & $123 \pm 7$ \\
\hline DBP $[\mathrm{mm} \mathrm{Hg}]$ & $97 \pm 5$ & $75 \pm 7$ \\
\hline
\end{tabular}


in Poznań, Poland) and involved 40 participants (17 females, 23 males; 39 to 65 years; see Table 3). The subjects were divided into control group (18 healthy people; 9 females, 9 males) and group of patients with newly diagnosed essential hypertension (22 patients; 8 females, 14 males).

The control group did not show any signs of organ's pathology (especially concerning cardiovascular system, liver and kidney's activity or inflammatory state) in subjective and biochemical examinations (blood morphology, ESR, lipid balance, liver tests, urine analysis) nor in additional examinations (blood pressure measurement). The questionnaire provided information about smoking history and medications.

In a pre-study period and during the study the participants did not take any drugs (or contraceptives in case of women). Nobody was abusing alcohol.

In both groups, a clinical study was conducted, including basic anthropometric measurement used to calculate BMI values. Weight was measured on a balance scale while height was measured in the standing position.

Blood pressure of all subjects was measured twice on the right arm after 5 minutes of rest, using a standard mercury sphygmomanometer. The mean of these 2 readings was used to classify blood pressure according to JNC VII, where hypertension is defined as systolic blood pressure more than $140 \mathrm{~mm} \mathrm{Hg}$ or diastolic blood pressure more than $90 \mathrm{~mm} \mathrm{Hg}$.

Secondary reasons of hypertension and obesity were excluded in the patients with newly diagnosed hypertension.

Patients with coexisting heart failure, ischemic heart disease, peripheral arteries disease (carotid arteries, vertebral arteries or lower limbs arteries), kidney failure (serum creatinine concentration > $115 \mathrm{nmol} / \mathrm{l}$ ), liver dysfunction (transaminases values 2.5 times higher than normal), diabetes (or disturbed tolerance to glucose) or acute/persistent inflammatory state were not qualified for the research.

The subjects were asked to fast for 10 hours. After all aseptic measures, $6 \mathrm{ml}$ of blood was collected from the antecubital vein while the subjects were sitting up right.

Serum homocysteine concentration was analyzed by immunochemical method with measurement of fluorescence intensity in polarized light (Fluorescence Polarization Immunoassay - FPIA) on IMx analyzer using ABBOTT commercial kits.

Written consent for participation in the study was a mandatory condition for taking part in the research.

\section{Statistical analyses}

The all statistical analyses were performed using the CSS STATISTICA program (V 7.0; StatSoft). The mean values and standard deviations were calculated using descriptive module. Before further analyses, normal distribution of the variables was checked with the Shapiro-Wilk test. Variables with abnormal distribution were analyzed by Mann-Whitney Test for comparisons within the groups. The statistical significance was determined at $p$ values below than 0.05 .

\section{Results}

The patients with essential hypertension had significantly higher homocysteine concentration (15.23 \pm $6.41 \mathrm{mmol} / \mathrm{L}$ vs. $9.71 \pm 3.21 ; \mathrm{p}=0.001$; see Table 4 and Figure 1) as compared to the control group; moreover, $28 \%$ of patients had the homocysteine level greater than $15 \mu \mathrm{mol} / \mathrm{l}$. Additionaly, the homocysteine

Table 4. Homocysteine concentration in patients and control group; * $p<0.05$, ${ }^{* *} p<0.001$

\begin{tabular}{ccc}
\hline Homocysteine concentration $[\mu \mathrm{mol} / \mathrm{l}]$ & Patients & Control \\
\hline Everyone & $15.23 \pm 6.41^{* *}$ & $9.71 \pm 3.21$ \\
\hline Smokers & $17.66 \pm 7.57^{*}$ & $10.56 \pm 3.18$ \\
\hline Non-smokers & $12.32 \pm 2.93$ & $8.63 \pm 3.12$ \\
\hline
\end{tabular}

Table 5. Correlation coefficients between homocysteine and studied parameters in patients with newly diagnosed essential hypertension and controls; ${ }^{*} p<0.05$

\begin{tabular}{ccc}
\hline & Homocysteine & \\
\hline Correlated parameters & Patients & Controls \\
\hline age & -0.09 & $0.68^{*}$ \\
\hline BMI & -0.16 & 0.31 \\
\hline SBP & -0.15 & 0.38 \\
\hline DBP & -0.12 & 0.36 \\
\hline
\end{tabular}




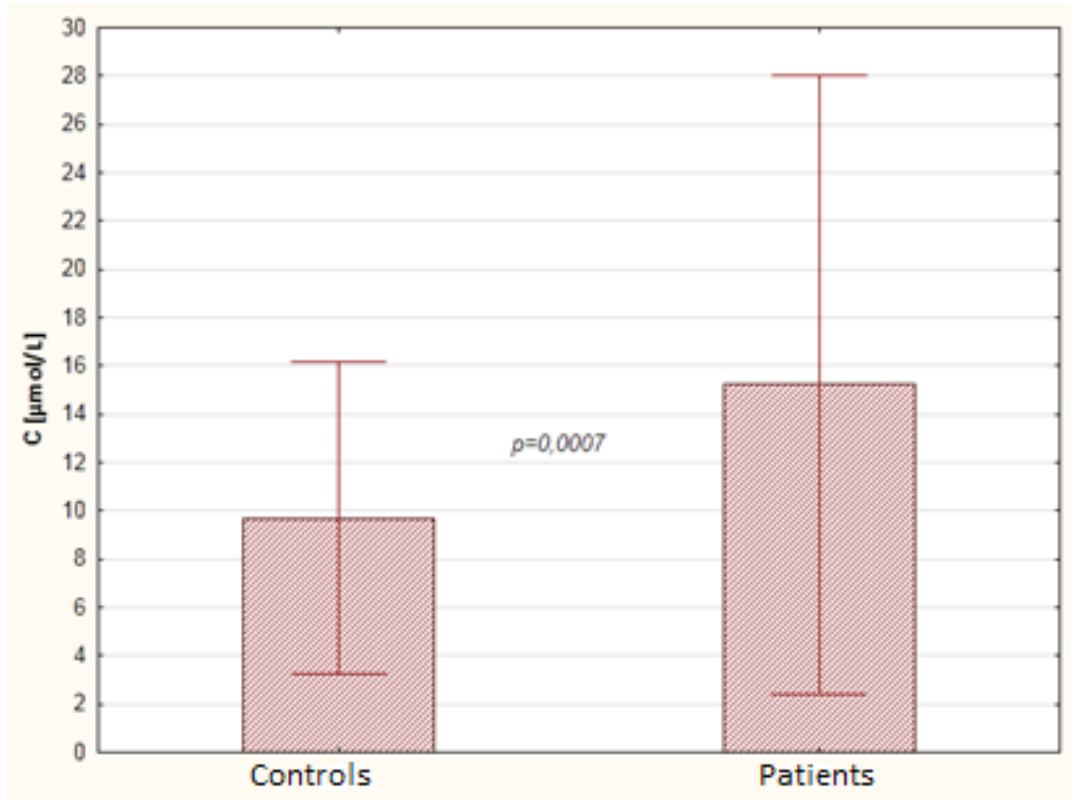

Figure 1. Blood homocysteine concentration in patients with newly diagnosed essential hypertension and controls

concentration in hypertensive smokers was significantly higher than in non-smokers ( $p=0.05$; see Table 4$)$.

No correlation was observed between homocysteine levels and age, diastolic and systolic blood pressure in patients with essential hypertension. In healthy volunteers only the correlation between age and homocysteine concentration was found. The results are summarized in Table 5.

\section{Discussion}

Homocysteine has been under a lot of speculation since its discovery in 1932. In 1969, an association between homocysteine and cardiovascular disease (CVD) was proposed when it was observed that people with a rare hereditary condition called homocystinuria are prone to develop severe cardiovascular disease in their teens and twenties. By the early 1990's, elevated homocysteine has been considered an independent risk factor for cardiovascular diseases (along with cholesterol and other lipid markers, age, gender, smoking status, obesity, hypertension and diabetes). It was shown that plasma homocysteine is more strongly associated with systolic than with diastolic blood pressure. As a result, it can increase arterial stiffness. However, the results of the studies investigating this hypothesis were inconsistent [13-15].

It is also believed that hyperhomocysteinemia damages endothelial cells, reduces the flexibility of vessels, and adversely affects the process of hemostasis. Additionaly, hyperhomocysteinemia increases the adverse effects of such risk factors as hypertension, smoking, impaired glucose, lipid and lipoprotein metabolism, and can promote the development of inflammation [7].

The meta-analysis performed by Boushey et al in 1995 indicated that an increase of $5 \mu \mathrm{mol} / \mathrm{L}$ was associated with a $60 \%$ increase in risk of coronary artery disease in men and an 80\% increase in risk in women. Also, a reported $50 \%$ increase in cerebrovascular disease was reported. This magnitude of increase in plasma homocysteine was thought to be equivalent to the CVD risk of a $19-\mathrm{mg} / \mathrm{dL}$ increase in cholesterol. The European Concerted Action Project also confirmed that the elevated plasma homocysteine was an independent risk factor for CVD, and calculated that an increase of 5 $\mu \mathrm{mol} / \mathrm{L}$ was associated with the increase in relative risk for CVD of 1.35. [16-17].

High blood pressure is a major risk factor for cardiovascular disease. It can be influenced by multiple non-hereditary risk factors (including dietetics and lifestyle). Among others, elevated plasma homocysteine may contribute to increase in blood pressure. Moreover, blood pressure may mediate part of the cardiotoxic effect of homocysteine [18].

Several epidemiological studies have examined the relationships between homocysteine and hypertension. Some of these examinations have found significant, although weak, association between plasma homocysteine and blood pressure. Elevated plasma Hcy levels have been consistently reported in hypertensive patients of different age and ethnicity. 
The results showed that fasting plasma homocysteine concentrations were significantly higher in subjects with hypertension than in those with normotension (mean +/- SEM, 8.1 +/- 0.6 v 6.8 +/- 0.2 micromol/L; $P<.05)$ [19]. It was shown that essential hypertension in adolescents is associated with lower folate and higher homocysteine levels, and with signs of insulin resistance. Therefore, hypertension in young individuals may be a part of early manifestation of insulin resistance syndrome, and that disturbed folate and homocysteine metabolism may play a role in early stages of hypertension [20-21].

The results of the National Health and Nutrition Examination Survey (NHANES) denoted that homocysteine was shown to have an independent, positive association with blood pressure, stronger in women than in men. A one-standard deviation increase in homocysteine was associated with an increase in systolic blood pressure (SBP) and diastolic blood pressure (DBP) of 0.7 and $0.5 \mathrm{~mm} \mathrm{Hg}$ in men, respectively, and in women, the increases in SBP and DBP were 1.2 and $0.7 \mathrm{~mm} \mathrm{Hg}$, respectively [8]. The comparison carried out by Sutton-Tyrrell et al reported that the level of plasma amino acid rose from $9.7 \mu \mathrm{mol} / \mathrm{L}$ at a SBP $<140 \mathrm{~mm} \mathrm{Hg}$ to $13 \mu \mathrm{mol} / \mathrm{L}$ at SBP $>180 \mathrm{~mm} \mathrm{Hg}$ [22]. Głowinska et al reported higher homocysteine level in hypertensive children [23].

Different results of this correlation were also obtained. Dinavahi et al. corroborated a significant, direct correlation of plasma homocysteine with SBP and DBP in premenopausal women, but not in men. However, when other factors, like age and body mass index were taken into account, no significant correlation was found [24].

Sundström et al. found no major relation of baseline plasma homocysteine level to hypertension incidence or longitudinal blood pressure progression in a large, community-based cohort of nonhypertensive individuals after adjustment for age, sex, and other important co-variates [25].

At vascular level, the continuing exposure to high homocysteine concentration leads to structural and functional changes in the vascular wall. Therefore, endothelial dysfunction seems to constitute a common association between homocysteine, hypertension and atherosclerosis.

There are some direct and indirect mechanisms by which high homocysteine exerts detrimental vascular effects. In a healthy endothelium NO rapidly reacts with homocysteine to form S-nitrosohomocysteine, which constitutes a protective mechanism. High homo- cysteine level can compromise NO bioavailability, inhibiting its regulatory endothelial vascular action thus leading to injury and dysfunction.

Increased homocysteine levels may also decrease NO bioavailability by increasing asymmetric dimethylarginine (ADMA) an analogue of L-arginine which acts as a competitive inhibitor of endothelial Nitric Oxide Synthase (eNOS). ADMA can also promote superoxide generation via uncoupling eNOS enzyme activity [26-27]. Reactive oxygen species (ROS)-induced oxidative stress represents a hallmark in endothelial dysfunction. A significant increase in reactive oxygen species at vascular levels in animal models and human hypertensive subjects has been described. Results of many studies strongly suggest that both processes, a diminished NO availability and increased ROS production coexist, constituting a common feature in human hypertension [28].

In hypertension, the increase in arterial wall thickness and the loss of elasticity over time results in the increase in pulse wave velocity, a direct measure of arterial stiffness. This change is reflected in gradual fragmentation and loss of elastin fibers and accumulation of stiffer collagen fibers in the media that occurs independently of atherosclerosis. Similar results are seen with an elevated level of homocysteine known as hyperhomocysteinemia, which increases vascular thickness, elastin fragmentation, and arterial blood pressure [29-30].

Cardiovascular diseases remain the main cause of mortality in industrialized countries and have become increasingly prevalent in developing countries. The risk of developing cardiovascular disease is mainly attributable to a number of known risk factors, which are in first instance hyperlipidemia, hypertension, smoking and diabetes mellitus. Smoking is one of the most important risk factors for cardiovascular diseases. Components of tobacco smoke cause physiological and morphological changes in endothelial cells and increase the concentration of many negatively acting substances, including homocysteine [31].

The association between elevated homocysteine concentrations and coronary, cerebral or peripheral artery disease was investigated in numerous epidemiological studies with either retrospective or prospective study design. The elevated homocysteine is known to increase oxidative stress that causes oxidative injury to the vascular endothelium, diminishes vasodilation by nitric oxide, stimulates the proliferation of vascular smooth muscle cells, and alters the elastic properties of the vascular wall. All these are associated with the 
rise in hypertension. Thus, homocysteine may contribute to blood pressure elevation. Higher levels of homocysteine in patients with primary hypertension may be an argument for introducing the evaluation of this amino acid concentration in clinical examinations.

It was observed that plasma homocysteine concentration in normotensive children of hypertensive parents is elevated before the development of hypertension. Therefore, homocysteine level may be predictive of the subsequent development of hypertension in such patients [32].

According to Catena et al., plasma homocysteine was significantly greater in hypertensive patients with evidence of carotid plaques than patients without carotid plaques. Moreover, carotid intima-media thickness progressively increased across quartiles of plasma Hcy levels and was independently related with age, blood pressure, C-reactive protein, and Hcy levels. These results suggest the role of elevated plasma homocysteine in the development and progression of carotid atherosclerosis [33].

\section{Conclusions}

1. Significantly higher homocysteine concentration was observed in the group of patients with primary hypertension, compared to the control group.

2. Significantly higher homocysteine concentration was observed in the group of smoking patients with primary hypertension, compared to the non-smoking patients.

3. No correlation was observed between homocysteine levels and age, diastolic, and systolic blood pressure in patients with essential hypertension.

\section{Acknowledgements}

\section{Conflict of interest statement}

The authors declare that there is no conflict of interest in the authorship or publication of contribution.

\section{Funding sources}

There are no sources of funding to declare.

\section{References}

1. Ganguly P, Alam SF. Role of homocysteine in the development of cardiovascular disease. Nutr J. 2015 Jan 10;14:6. doi: 10.1186/1475-2891-14-6.

2. Atif A, Rizvi MA, Tauheed S, Aamir I, Majeed F, Siddiqui $K$, Khan S. Serum homocysteine concentrations in patients with hypertension. Pak J Physiol. 2008;4(1): 21-22.

3. Marinou K, Antoniades C, Tousoulis D, Pitsavos C, Goumas G, Stefanadis C. Homocysteine: a risk factor for coronary artery disease? Hellenic J Cardiol. 2005;46(1):59-67.
4. Faeh D, Chiolero A, Paccaud F. Homocysteine as a risk factor for cardiovascular disease: should we (still) worry about it? Swiss Med Wkly. 2006;136:745-756.

5. Leal AA, Palmeira AC, Castro GM, Simões MO, Ramos AT, Medeiros CC. Homocysteine: cardiovascular risk factor in children and adolescents? Rev Assoc Med Bras. 2013;59(6):622-628.

6. Giannoni M, Consales V, Campanati A, Ganzetti G, Giuliodori K, Postacchini $V$ et al. Homocysteine plasma levels in psoriasis patients: our experience and review of the literature. J Eur Acad Dermatol Venereol. 2015 Mar 23. doi: 10.1111/jdv.13023. [Epub ahead of print]

7. Baszczuk A, Kopczynski Z, Thielemann A. Endothelial dysfunction in patients with primary hypertension and hyperhomocysteinemia. Postepy Hig Med Dosw 2014; 68:91-100.

8. Lim U, Cassano PA. Homocysteine and Blood Pressure in the Third National Health and Nutrition Examination Survey, 1988-1994. Am J. Epidemiol. 2002;156(12): 1105-1113.

9. Sen U, Tyagi SC. Homocysteine and Hypertension in Diabetes: Does PPAR $\gamma$ Have a Regulatory Role? PPAR Res. 2010; 2010: 806538.

10. Vermeulen EG, Niessen HW, Bogels $M$, Stehouwer $C D$, Rauwerda JA., van Hinsbergh VW. Decreased smooth muscle cell/extracellular matrix ratio of media of femoral artery in patients with atherosclerosis and hyperhomocysteinemia. Arterioscler Thromb Vas Biol 2001;21(4):573-577.

11. Stehouwer CD, van Guldener C. Does homocysteine cause hypertension? Clin Chem Lab Med. 2003;41(11): 1408-1411.

12. van Guldener CP, Nanayakkara PW, Stehouwer CD. Homocysteine and blood pressure. Current Hypertension Reports. 2003;5(1):26-31.

13. Nakhai-Pour HR, Grobbee DE, Bots ML, Muller M, van der Schouw YT. Circulating homocysteine and large arterial stiffness and thickness in a population-based sample of middle-aged and elderly men. J Hum Hypertens. 2007;21:942-948.

14. Ruan L, Chen W, Srinivasan SR, Xu J, Sun M, Toprak A, Berenson GS. Relation of plasma homocysteine to arterial stiffness in black and white young adults (from the Bogalusa Heart Study) Am J Cardiol. 2009;103:985-988.

15. Levy D, Hwang SJ, Kayalar A, Benjamin EJ, Vasan RS, Parise $\mathrm{H}$ et al. Associations of plasma natriuretic peptide, adrenomedullin, and homocysteine levels with alterations in arterial stiffness: the Framingham Heart Study. Circulation. 2007;115:3079-3085.

16. Boushey CJ, Beresford SA, Omenn GS, Motulsky AG. A quantitative assessment of plasma homocysteine as a risk factor for vascular disease. Probable benefits of increasing folic acid intakes. JAMA 1995;274:1049-1057.

17. Graham IM, Daly LE, Refsum HM, Robinson K, Brattström LE, Ueland PM et al. Plasma homocysteine as a risk factor for vascular disease. The European Concerted Action Project. JAMA. 1997;277:1775-1781.

18. Animesh K, Mehrotra V. Trends in blood pressure with increasing plasma homocysteine levels. JIACM 2014; 15(3-4):188-191.

19. Sheu WH, Lee WJ, Chen YT. Plasma homocysteine concentration and insulin sensitivity in hypertensive subjects. Am J Hypertens. 2000;13(1 Pt 1):14-20. 
20. Kahleova R, Palyzova D, Zvara K, Zvarova J, Hrach K, Novakova I, et al. Essential hypertension in adolescents: association with insulin resistance and with metabolism of homocysteine and vitamins. Am J Hypertens 2002;15(10 Pt 1):857-864

21. Ustundag S, Arikan E, Sen S, Esgin H, Ciftci S. The relationship between the levels of plasma total homocysteine and insulin resistance in uncomplicated mild-to-moderate primary hypertension. J Hum Hypertens, 2006;20(5):379-81.

22. Sutton-Tyrrell K, Bostom A, Selhub J, Zeigler-Johnson C. High homocysteine levels are independently related to isolated systolic hypertension in older adults. Circulation. 1997:96:1745-1749.

23. Głowinska B, Urban M, Koput A, Galar M. New atherosclerosis risk factors in obese, hypertensive and diabetic children and adolescents. Atherosclerosis. 2003;167:275286.

24. Dinavahi R, Cossrow N, Kushner H, Falkner B. Plasma homocysteine concentration and blood pressure in young adult African Americans. Am J Hypertens. 2003;16:767770.

25. Sundström J, Sullivan L, D'Agostino RB. Plasma Homocysteine, Hypertension Incidence, and Blood Pressure Tracking.The Framingham Heart Study. Hypertension. 2003;42:1100.

26. Stanger $\mathrm{O}$, Weger M. Interactions of homocysteine, nitric oxide, folate and radicals in the progressively damaged endothelium. Clin Chem Lab Med 2003;41:1444-1454.

27. Rodriguez-Esparragon F, Caballero-Hidalgo A, Hernandez-Trujillo Y, Macias-Reyes A, Rodriguez-Perez JC. Concerning Arterial Hypertension, Homocysteine and Paraoxonase-1. Current Hypertension Reviews. 2007;3:51-57.
28. Touyz RM. Reactive oxygen species, vascular oxidative stress, and redox signaling in hypertension: what is the clinical significance? Hypertension 2004; 44: 248-252.

29. Rodrigo R, Passalacqua W, Araya J, Orellana M, Rivera G. Homocysteine and EssentialHypertension. J Clin Pharmacol December 2003: 43 (12):1299-1306.

30. Steed MM, Tyagi SC. Mechanisms of Cardiovascular Remodeling in Hyperhomocysteinemia Antioxid Redox Signal.2011;15(7):1927-1943.

31. O'Callaghan P, Meleady R, Fitzgerald T, Graham I. Smoking and plasma homocysteine. European Heart Journal 2002; 23: 1580-1586.

32. Yıldırım A, Keleş F, Özdemir G, Koşger P, Uçar B, Alataş Ö, Kılıç Z. Homocysteine levels in normotensive children of hypertensive parents. Anatol J Cardiol. 2015 Apr 2. doi: 10.5152/akd.2015.5862. [Epub ahead of print]

33. Catena C, Colussi G, Url-Michitsch M, Nait F, Sechi LA. Subclinical carotid artery disease and plasma homocysteine levels in patients with hypertension. J Am Soc Hypertens. 2015 Mar;9(3):167-75.

Acceptance for editing: 2015-04-29 Acceptance for publication: 2015-05-28

Correspondence address: Department of Clinical Pharmacology Poznan University of Medical Sciences Długa 1/2 Str., 61-848 Poznań, Poland phone: +48 618533161 e-mail: artcies@gmail.com 